\title{
Simulation of Partial and Supercavitating Flows around Axisymmetric and Quasi-3D Bodies by Boundary Element Method Using Simple and Reentrant Jet Models at the Closure Zone of Cavity
}

\author{
M. Nouroozi, M. Pasandidehfard, and M. H. Djavareshkian \\ Ferdowsi University of Mashhad, P.O. Box 91775-1111, Mashhad, Iran \\ Correspondence should be addressed to M. Pasandidehfard; fard_m@um.ac.ir
}

Received 3 February 2016; Accepted 10 April 2016

Academic Editor: Song Cen

Copyright (C) 2016 M. Nouroozi et al. This is an open access article distributed under the Creative Commons Attribution License, which permits unrestricted use, distribution, and reproduction in any medium, provided the original work is properly cited.

A fixed-length Boundary Element Method (BEM) is used to investigate the super- and partial cavitating flows around various axisymmetric bodies using simple and reentrant jet models at the closure zone of cavity. Also, a simple algorithm is proposed to model the quasi-3D cavitating flows over elliptical-head bodies using the axisymmetric method. Cavity and reentrant jet lengths are the inputs of the problem and the cavity shape and cavitation number are some of the outputs of this simulation. A numerical modeling based on Navier-Stokes equations using commercial CFD code (Fluent) is performed to evaluate the BEM results (in 2D and $3 \mathrm{D}$ cases). The cavitation properties approximated by the present research study (especially with the reentrant jet model) are very close to the results of other experimental and numerical solutions. The need for a very short time (only a few minutes) to reach the desirable convergence and relatively good accuracy are the main advantages of this method.

\section{Introduction}

The cavitating flows around various bodies have been the subject of extensive theoretical, numerical, and experimental research in recent decades. When the pressure of liquid becomes equal to or less than its saturated vapor pressure in fixed temperature conditions, the balance of fluid intermolecular forces is disrupted and subsequently a tensile stress occurs. The tensile stress leads to the development of vapor cavities in the liquid. Formation and growth of cavity usually occur when the liquid is subjected to sudden changes of pressure. This event can happen in many fluid systems like pumps, nozzles, turbine blades, and hydrofoils. In these systems, cavitation is known for its destructive effects like noise production, corrosion, and reduction of efficiency; therefore, researchers and engineers attempt to minimize these destructive effects. On the other hand, in some applications, cavitation can be used as a beneficial phenomenon. For example, in high-speed submerged vehicles, cavitation is desired since it leads to a significant reduction of drag force. This advantage of cavitation phenomenon is exploited to increase velocity and efficiency [1].

It has been observed that the main fluid flow passing over the cavity surface tends to return into the cavity from the end of it. This flow is called "reentrant jet." Major reason for developing reentrant jet is a tendency of the fluid to move from the higher pressure zone (on the cavity surface) to the lower pressure zone (inside the cavity). Reentrant jet is developed under specific conditions. These conditions include (a) a high reverse pressure gradient at the closure of the cavity and (b) a considerable thickness of the cavity. Cavities of extremely large length or very small length do not satisfy (a) and (b) conditions, respectively. Therefore, smalland large-length cavities do not have a considerable reentrant jet, and only medium-length cavities are accompanied by reentrant jet [2]. Due to the various flow directions and phases at the end of the cavity, simulation of this zone is very complicated. In contrast to Navier-Stokes equations which do not require the end of cavity to be simulated, this zone should be modeled in BEM. Several models have been suggested for 
the cavity closure. In the present study, two models have been used for this critical zone of the cavity: simple closure model and reentrant jet model. In simple closure model, the cavity is closed on the body surface and consequently a stagnation point is formed at the end of the cavity. In the reentrant jet model, the flow over the cavity surface changes its direction into the cavity. The reentrant jet velocity is typically assumed to be of the order of the velocity of the flow over cavity surface. Reentrant jet closure model closely fits the behavior of unsteady cavitating flows.

BEM has potential flow as a basic assumption. As a result, one can use BEM for cavitation analysis only when cavitating flow is proven to be potential. Experimental studies of Labertaux and Ceccio show that the flow around cavity is reasonably approximately a potential one [3]. Therefore, potential theories (such as BEM) may be utilized for the simulation of cavity around bodies. BEM is based on "Green theory." Green theory states that every incompressible and irrotational flow can be simulated by source, dipole, or vortex distribution on its boundary surfaces [4]. In the present work, dipole and source rings have been distributed on the body/cavity boundaries to simulate cavitating flows over axisymmetric geometries.

The first studies for cavitation were carried out by Efros [5] and Gilbarg and Serrin [6] using 2D analytical theory of free streamlines. After that, modeling of cavitation over hydrofoils using the linear theory of flow was introduced. Tulin [7, 8] and Guerst [9] developed this method. After Hess and Smith [10], who calculated potential flow around arbitrary bodies, the application of this method rapidly increased. For the first time, Uhlman [11] used a nonlinear BEM based on velocity to solve the $2 \mathrm{D}$ partial cavitating flows around hydrofoil using a vortex distribution over the flow boundaries. They proceeded to use the same method to solve the 2D supercavitating flow around hydrofoil [12]. Kinnas and Fine [13] offered another nonlinear BEM based on potential to solve partial cavitating flow on 2D hydrofoil. They started solving this problem by distributing source and dipole on the boundaries of the flow using Green's third identity. The convergence and accuracy of potential-based BEM were better than those of the velocity-based BEM. After that, Birkhoff and Zarantonello [14] and Gilbarg [15] investigated formation of a reentrant jet of the cavity in symmetric flow against a vertical flat plate for the first time; other researchers such as Pellone and Rowe [16], Fine and Kinnas [17], and Vaz [18] employed the reentrant jet model for the cavity termination. Also, Uhlman [19], Nouri et al. [20], and Rashidi et al. [21] used potential-based BEM for cavitating flows over axisymmetric bodies. Uhlman et al. used this method for supercavitating flows using reentrant jet model and Nouri et al. solved these flows using simple closure model. Pasandideh-fard et al. applied the method together with simple closure model for partial and supercavitating flows.

In this work, a BEM analysis of partial and supercavitating flows around axisymmetric bodies is presented and two models of termination of the cavity (simple model and reentrant jet) are compared. To simulate these flows, body, cavity, and jet boundaries are approximated by elements and source and dipole rings are distributed on them, based on Green's third identity. In addition, modeling of cavitating flows is performed with zero angle of attack over quasi3D bodies (cylinders with elliptical head) using present axisymmetric code. To evaluate the results, $2 \mathrm{D}$ and $3 \mathrm{D}$ numerical commercial CFD codes (Fluent) are executed. The present work, in a novel manner, employs the reentrant jet model at the end of partial cavity over different axisymmetric geometries. Besides, the main innovation of this research is the exploitation of the rapid convergence capability of axisymmetric BEM in the modeling of cavitating flows over elliptical-head cylinders (quasi-3D flow). This algorithm had not been reported in the literature. It is noteworthy that the present quasi-3D analysis yields relatively appropriate results at minimal computational and time cost (only in a few minutes), whereas the other numerical methods involve greater complication and much higher cost (they take at least hours).

\section{Mathematical Equations}

The cylindrical coordinate system is the best coordinate system for axisymmetric flows. Integral expression of Green's third identity in this coordinate system is as follows:

$$
\begin{aligned}
& 2 \pi \varphi(r, x) \\
& \quad=\oiint_{S}\left\{\frac{\partial \varphi}{\partial n} G(x, r ; \zeta, \rho)-\varphi(r, x) \frac{\partial G(x, r ; \zeta, \rho)}{\partial n}\right\} \\
& \quad \cdot \rho d \varphi d s,
\end{aligned}
$$

where $n$ is the normal vector directed outward from the solidbody surface and the cavity interface, $s$ is arc length along a meridian, $x$ and $r$ are the components of the axisymmetric coordinate system, and $\varphi$ is "disturbance" potential on the solved surfaces. In fact, disturbance potential, $\varphi$, is the sum of all the potential flow elements, except free stream potential, in one point [11]. Thus, the total $(\phi)$ and disturbance $(\varphi)$ potentials are related by

$$
\phi=U_{\infty} \cdot x+\varphi
$$

where $U_{\infty}$ is the free stream velocity that flows on the body surface. Considering $U_{\infty}$ to be equal to unit, (2) becomes dimensionless:

$$
\phi=x+\varphi .
$$

Since the bodies are axisymmetric, distributed potential elements on the body/cavity surfaces should be rings to be appropriate for the cylindrical coordinate system. $G$ and $\partial G / \partial n$ are the potential functions related to the sources and dipoles distributed around a ring, respectively [3]. Source and dipole rings used in Green theory can be obtained by integrating these potential elements around the axis [19]. The potential function of a source ring $(G)$ with radius $\rho$ at point $(x, r)$, when the ring center is located at $\zeta$ and for the unit surface of $s$, is defined as follows:

$$
G(x, r ; \zeta, R)=\int_{-\pi}^{+\pi} \frac{\rho d \varphi}{\sqrt{(x-\zeta)^{2}+r^{2}+R^{2}-2 r R \cos \theta}} .
$$


And the potential function of a dipole ring $(\partial G / \partial n)$ that is the normal derivative of the source ring is defined as follows:

$$
\begin{aligned}
& \frac{\partial}{\partial n} G(x, r ; \zeta, R) \\
& \quad=\int_{-\pi}^{+\pi}\left\{\frac{1}{\sqrt{(x-\zeta)^{2}+r^{2}+R^{2}-2 r R \cos \theta}}\right\} \rho d \varphi \\
& \quad=\int_{-\pi}^{+\pi}\left\{\zeta_{n} \frac{\partial G}{\partial \zeta}+\rho_{n} \frac{\partial G}{\partial \rho}\right\} \rho d \varphi .
\end{aligned}
$$

Cylindrical coordinate components used in (4) and (5) are shown in Figure 1.

\section{Boundary Conditions}

3.1. Kinematic Boundary Condition on the Body/Cavity Surface. The surface impenetrability condition requires flow to have no normal component on the body surface. In addition, flow is assumed to have no normal component on the cavity surface. It means that flow on the body/cavity surface has only the tangent component. Therefore, kinematic boundary condition on the body $\left(S_{b}\right)$ and cavity $\left(S_{c}\right)$ surfaces in disturbance potential form is

$$
\frac{\partial \varphi}{\partial n}=-x_{n} \quad \text { on } S_{b} \cup S_{c},
$$

where $x_{n}$ is unit vector normal to the body/cavity surface. To satisfy the kinematic condition on the cavity surface, normal component of velocity on the cavity surface should be zero. Therefore, the slope of the velocity vector should be equal to the slope of the cavity boundary at every point on it. Physically, this condition means that there is no entering/exit flow to/from the cavity. The kinematic condition on the cavity surface is not employed in the integral equations that form the linear equation system. It is used to correct the position of the cavity elements in successive iterations. In fact, the problem becomes a convergent one when the kinematic boundary condition is satisfied on the cavity.

3.2. Dynamic Boundary Condition on the Cavity Surface. The flow around the cavity is irrotational. As a result, a constant velocity of $U_{\infty} \sqrt{1+\sigma}$ may be obtained for the flow on the cavity surface by using the Bernoulli equation, in which $\sigma$ is the cavitation number and is defined as follows:

$$
\sigma=\frac{P_{\infty}-P_{c}}{(1 / 2) \rho U_{\infty}^{2}}
$$

where $U_{\infty}$ and $P_{\infty}$ denote free stream velocity and pressure, respectively $[2,17-20] . P_{c}$ is the vapor pressure of liquid. The tangential velocity component on the cavity surface in disturbance potential form is given by [17-20]:

$$
\frac{\partial \varphi}{\partial s}=\sqrt{1+\sigma}-x_{s} \text { on } S_{c}
$$

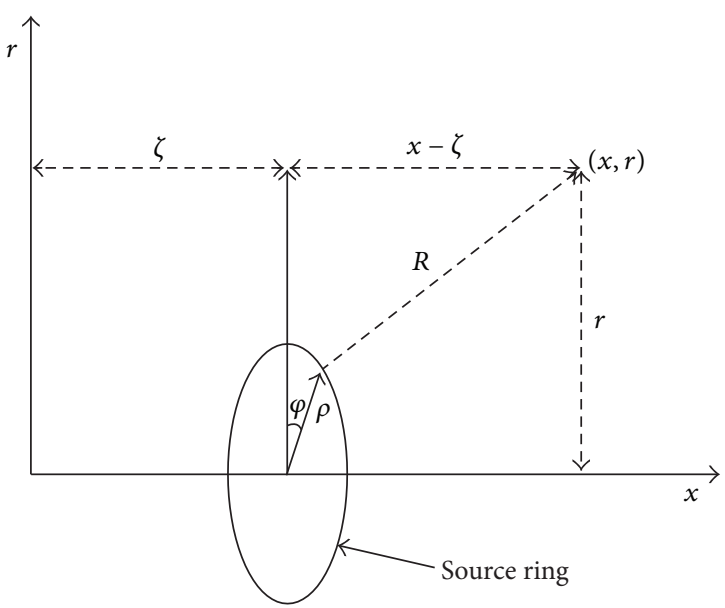

FIGURE 1: Cylindrical coordinate components used in Green equations.

Integrating (8) results in the following for the values of the dipoles distribution on the cavity:

$$
\varphi=\varphi_{0}+\sqrt{1+\sigma}\left(s-s_{0}\right)-\left(x-x_{0}\right) \text { on } S_{c},
$$

where $s$ is the unit vector tangent to the cavity boundary and $\varphi_{0}$ is the potential of dipole at the detachment point of the cavity. The dynamic boundary condition ensures that the pressure on the cavity is constant and equal to the vapor pressure.

3.3. Dynamic Boundary Condition on the Reentrant Jet. Experimental data verify that reentrant jet velocity is of the order of the magnitude of the free stream velocity although it can be changed by the cavity thickness [22]. Based on the potential flow analysis, it is hypothesized that reentrant jet returns into the cavity with a constant thickness, and the normal velocity component of the reentrant jet is considered to be equal to the flow velocity on the cavity boundary, that is, $\sqrt{1+\sigma}$ [19]. Therefore, the dynamic boundary condition on the reentrant jet in disturbance potential form is

$$
\frac{\partial \varphi}{\partial n}=\sqrt{1+\sigma}-x_{n} \quad \text { on } S_{j} .
$$

The constant potential on the reentrant jet surface is calculated by integrating (10):

$$
\varphi=\varphi_{0}+\sqrt{1+\sigma}\left(s_{j}-s_{0}\right)-\left(x_{j}-x_{0}\right) \text { on } S_{j} \text {, }
$$

where $s_{j}$ and $x_{j}$ are arc length and $x$ coordinate at the edge of the jet cross section, respectively. The various boundary conditions satisfied on the body, cavity, and reentrant jet surfaces are shown in Figure 2.

\section{Governing Integral Equations}

The potential of each dipole $(\varphi)$ on the body surface and the potential of each source $\partial \varphi / \partial n$ on the cavity surface are unknown (1). According to the abovementioned boundary 
conditions, the cavitation number being known, $\varphi$, on the cavity and reentrant jet is calculated from (9) and (11), and $\partial \varphi / \partial n$ on the body and reentrant jet is calculated from (6) and (10). Placing the unknowns on the left-hand side and the knowns on the right-hand side, Green's third identity for the wetted portion of the solid-body/cavity boundary is written as follows:

$$
\begin{aligned}
& 2 \pi \varphi+\iint_{S_{b}} \varphi \frac{\partial G}{\partial n} d S-\iint_{S_{c}} \frac{\partial \varphi}{\partial n} G d S \\
&=\iint_{S_{b}+S_{j}} \frac{\partial \varphi}{\partial n} G d S-\iint_{S_{c}+S_{j}} \varphi \frac{\partial G}{\partial n} d S \text { on } S_{b} .
\end{aligned}
$$

And for the cavity interface, it is written as follows:

$$
\begin{aligned}
& \iint_{S_{b}} \varphi \frac{\partial G}{\partial n} d S-\iint_{S_{c}} \frac{\partial \varphi}{\partial n} G d S \\
& \quad=\iint_{S_{b}} \frac{\partial \varphi}{\partial n} G d S-2 \pi \varphi-\iint_{S_{c}} \varphi \frac{\partial G}{\partial n} d S \text { on } S_{c} .
\end{aligned}
$$

Implementing the above boundary conditions, (12) on the solid body can be written as follows:

$$
\begin{aligned}
2 \pi \varphi+ & \iint_{S_{b}} \varphi \frac{\partial G}{\partial n} d S-\iint_{S_{c}} \frac{\partial \varphi}{\partial n} G d S+\varphi_{0} \iint_{S_{c}+S_{j}} \frac{\partial G}{\partial n} d S \\
& +\sqrt{1+\sigma}\left[\iint_{S_{c}+S_{j}}\left(s-s_{0}\right) \frac{\partial G}{\partial n} d S-\iint_{S_{j}} G d S\right] \\
= & -\iint_{S_{b}} x_{n} G d S-\iint_{S_{c}+S_{j}}\left(x-x_{0}\right) \frac{\partial G}{\partial n} d S \\
& -\iint_{S_{j}} x_{n} G d S \text { on } S_{b} .
\end{aligned}
$$

Equation (13) on the cavity interface becomes

$$
\begin{aligned}
& \iint_{S_{b}} \varphi \frac{\partial G}{\partial n} d S-\iint_{S_{c}} \frac{\partial \varphi}{\partial n} G d S+\varphi_{0}[2 \pi \\
& \left.\quad+\iint_{S_{c}+S_{j}} \frac{\partial G}{\partial n} d S\right]+\sqrt{1+\sigma}\left[2 \pi\left(s-s_{0}\right)\right. \\
& \left.\quad+\iint_{S_{c}+S_{j}}\left(s-s_{0}\right) \frac{\partial G}{\partial n} d S-\iint_{S_{j}} G d S\right] \\
& \quad=-\iint_{S_{b}} x_{n} G d S+\left[2 \pi\left(x-x_{0}\right)\right. \\
& \left.\quad+\iint_{S_{c}+S_{j}}\left(x-x_{0}\right) \frac{\partial G}{\partial n} d S\right]-\iint_{S_{j}} x_{n} G d S \text { on } S_{c} .
\end{aligned}
$$

In addition to these equations, an auxiliary condition is required to match the number of equations ( $N_{b}$ equations on the body $+N_{c}$ equations on the cavity + an auxiliary equation) and unknowns $\left(N_{b}\right.$ dipole strength on the body $+N_{c}$ source

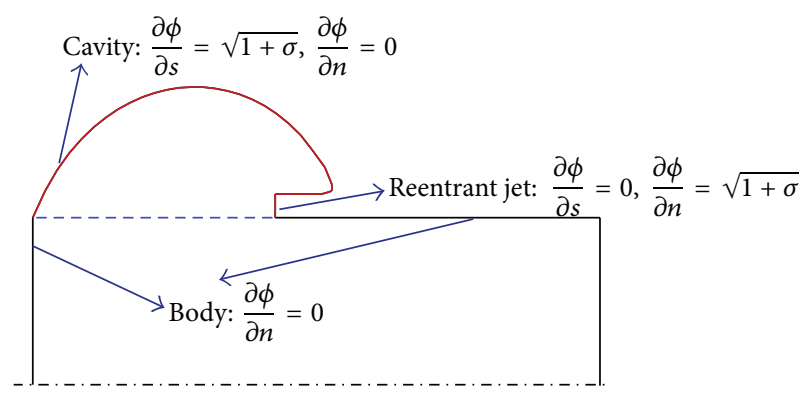

FIGURE 2: Illustration of various boundary conditions on the surfaces of solution.

strength on the cavity + cavitation number) in the system of linear equations. In order to do this, we impose the concept that the net strength of the sources distributed on the cavity boundaries is equal to the flux through the jet, which may be expressed as follows:

$$
\iint_{S_{c}} \frac{\partial \varphi}{\partial n} d S=\iint_{S_{b}} x_{n} d S .
$$

To impose these equations on the cavitation problem, the wetted body, cavity, and reentrant jet surfaces are approximated by linear elements as an initial shape. Applying (14) and (15) on the wetted body and cavity elements, respectively, and using (16) will build a system of linear equations. Using Gaussian elimination to solve this system of linear equations, the strengths of the dipoles and sources and the cavitation number are calculated.

\section{Geometry}

5.1. Body. In this work, partial cavitation has been simulated using BEM over several cylindrical bodies with different cavitator shapes: hemispherical, blunt cylindrical, and conical nose shapes (with half-cone angle of 40 degrees) and supercavitation has been modeled over a disk cavitator. Longitudinal quantities have been nondimensionalized with the diameter of the cylinder forehead.

5.2. Cavity. In BEM, the detachment and end points of the cavity are the inputs of the problem. As experimentally observed by Washio et al. [23], in the geometries with an edge, such as blunt and conical cylinders and disk cavitator, cavity starts from the fracture point (forehead edge), and in the smooth bodies, such as hemispherical cylinder, cavity starts from the separation point of the flow. Considering that the cavity length is an input to the problem, once the detachment point is known, the end point of the cavity will subsequently be specified. Since the cavity elements change their position in every iteration, these ones are considered finer than other elements.

5.3. Reentrant Jet. Suppose that the reentrant jet thickness is constant. In order to determine the reentrant jet thickness, 
the following analytic relation, which was proposed by Callenaere et al. [22], is used:

$$
\lambda=\frac{1}{2}\left[1-\frac{1}{\sqrt{1+\sigma}}\right]+\frac{1}{4} \frac{d C_{P}}{d(x / l)} d .
$$

It is composed of the cavitation number $(\sigma)$, pressure coefficient gradient $\left(C_{P}\right)$ at the cavity closure, maximum value of the cavity thickness $(d)$, and the cavity length $(l)$. Indeed, the first part of (17) represents the effect of the cavitation number on the reentrant jet thickness and its second part denotes the effect of the pressure gradient at the cavity closure. The reentrant jet thickness is calculated to be less than 30 percent of the maximum value of the cavity thickness. This is consistent with the other studies [22].

In this work, reentrant jet length is a fraction of cavity length. In all cases, the reentrant jet length is assumed to be half of the cavity length. It will be demonstrated that the solution is essentially independent of the length chosen for the reentrant jet.

\section{Quasi-3D Algorithm}

When the flow with zero angle of attack (axisymmetric income flow) moves around an elliptical-head cylinder (nonaxisymmetric body), a quasi-3D flow is formed. The axisymmetric BEM equation is utilized to simulate the cavitating flow around quasi-3D flows. The relevant algorithm for this solution is as follows: once the ellipse aspect ratio, the ratio of the larger diameter to the smaller one, is determined, the elliptical head of the cavitator is segmented and, for each segment, axisymmetric BEM is implemented and converged as mentioned above. Indeed, the proposed quasi-3D solution is a superposition of the axisymmetric solutions for the various segments of the elliptical head, as shown in Figure 3. For the quasi-3D solution to converge, all segments should converge to the same cavitation number. The analysis is dimensionless and there is a one-to-one correspondence between cavitation numbers and dimensionless cavity lengths; therefore, for any given cavitation number, the ratio of the cavity length to the diameter of the corresponding segment is a fixed value. As a result, knowing the diameter of a given cavitator segment, the corresponding cavity length may readily be calculated and employed as an input to BEM. Consequently, the partial cavity shape is determined at the various sections of the elliptical-head cavitator for a specified cavitation number. As lateral flows are negligible for zero angle of attack flows, it is justifiable to use axisymmetric equations for any segment of quasi-3D geometries. As mentioned in the Introduction, this algorithm has the benefit of quick convergence with an acceptable accuracy and easy meshing in the analysis of cavitating flows.

\section{Numerical Method}

In the present study, in order to validate the result from BEM, a multiphase mixture method is used to analyze axisymmetric steady partial cavitating flow over a blunt-head cylinder for the cavitation numbers in the range of $0.15-0.5$ using

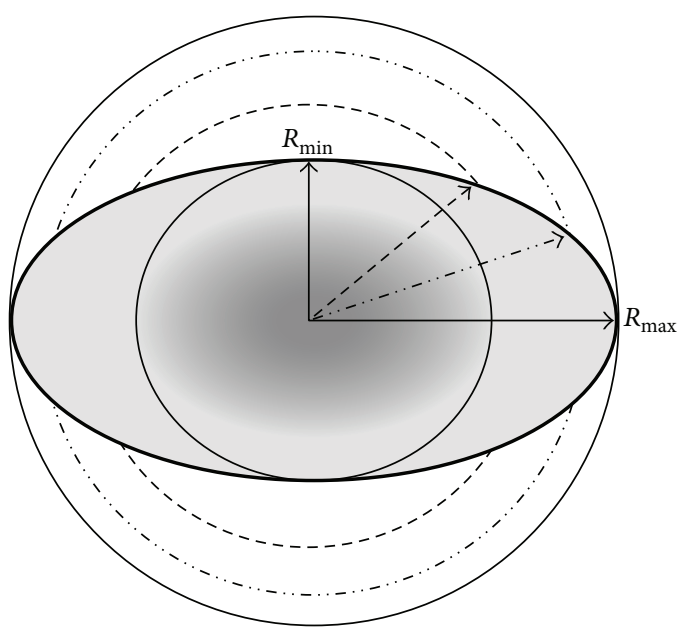

FIGURE 3: Superposition of the axisymmetric solutions (circle-head sections) for the various segments of the elliptical-head section.

commercial CFD code (Fluent). The cylinder is set to be 20 units long (nondimensionalized by the diameter of the cylinder cross section) and is surrounded by a uniform flow of water at the range of $20-46.33 \mathrm{~m} / \mathrm{s}$. In this research, a pressure-based implicit procedure is used to solve the NavierStokes equations. The method employs an iteration technique in which the different conservation equations (continuity and momentum) are solved successively. The present work employs the Simple technique in which the implicit discretized equations are solved. In addition, a homogeneous mixture model is used to model multiphase flow [24, 25]. The problem is analyzed using appropriate boundary conditions and structured meshing (with nearly 92000 meshes in the axisymmetric modeling and 600000 meshes in the 3D one). At the inlet and the upper walls of domain (and also the lower walls for 3D case), velocity has been prescribed. At the outlet, the pressure is fixed and wall boundary conditions are used for body surface. For axisymmetric case, symmetry boundary conditions are used on symmetry line of domain. To ensure that the solution is independent of the computational zone, the height of the zone above the axisymmetric shape is assumed to be $70 R_{\text {cavitator }}$, the length of the inlet flow (at the front of the cylinder) to be $50 R_{\text {cavitator }}$, and the length of the outlet flow (at the back of the cylinder) to be $60 R_{\text {cavitator }}$ Also, to ensure that the solution is independent of the meshing, the near-shape zone, that is, where the cavity is formed, is very much finely meshed. In Figure 4, the diagram for the independence of the solution from the structured meshing is shown.

\section{Results and Discussions}

8.1. Supercavitation over Axisymmetric Bodies. Figures 5(a) and 5(b) demonstrate how increasing the number of elements on the body/cavity boundaries affects the convergence of BEM. In Figures 6(a) and 6(b), the cavity shapes over a disc, in $2 \mathrm{D}$ and $3 \mathrm{D}$ views, as predicted by BEM are shown for the exemplary cavity lengths of 5 and 10. The proposed method 


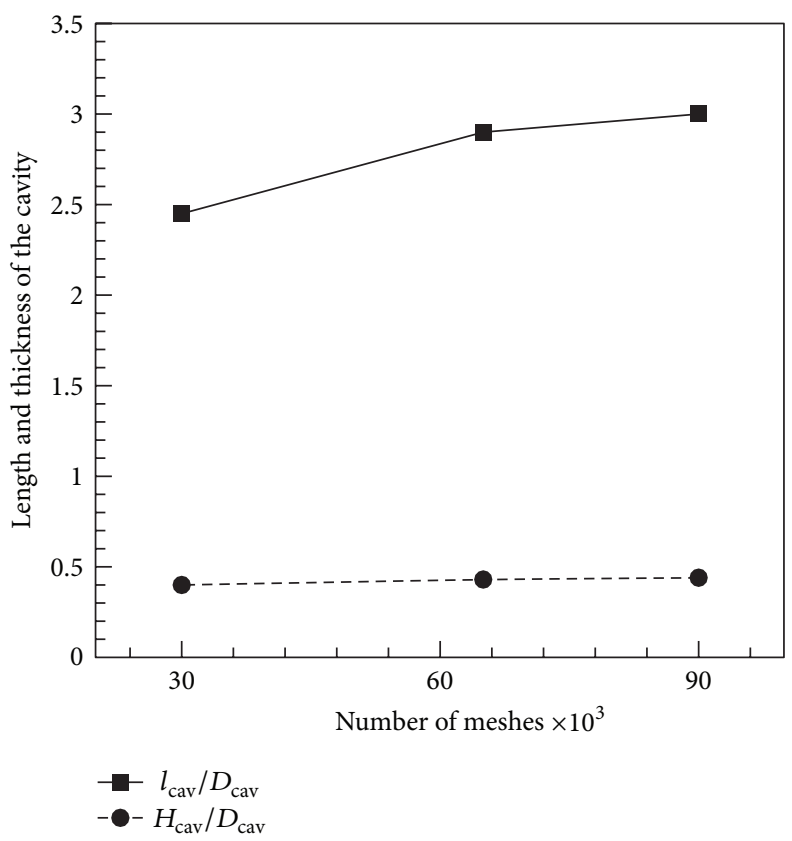

Figure 4: Convergence of the dimensionless length $\left(l_{\text {cav }} / D_{\text {cav }}\right)$ and thickness $\left(H_{\text {cav }} / D_{\text {cav }}\right)$ of the cavity computed by Fluent code with increasing number of meshes in the computational zone for the axisymmetric steady partial cavitating flow over a blunt-head cylinder $(\sigma=0.3)$.

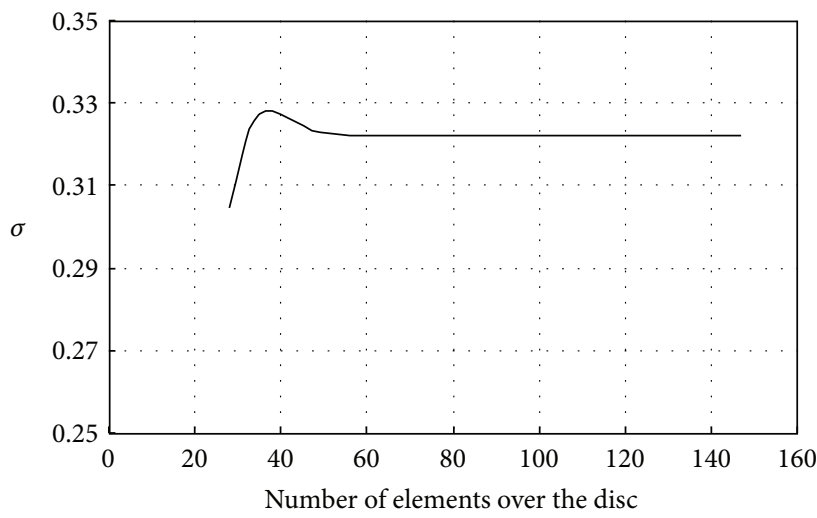

(a)

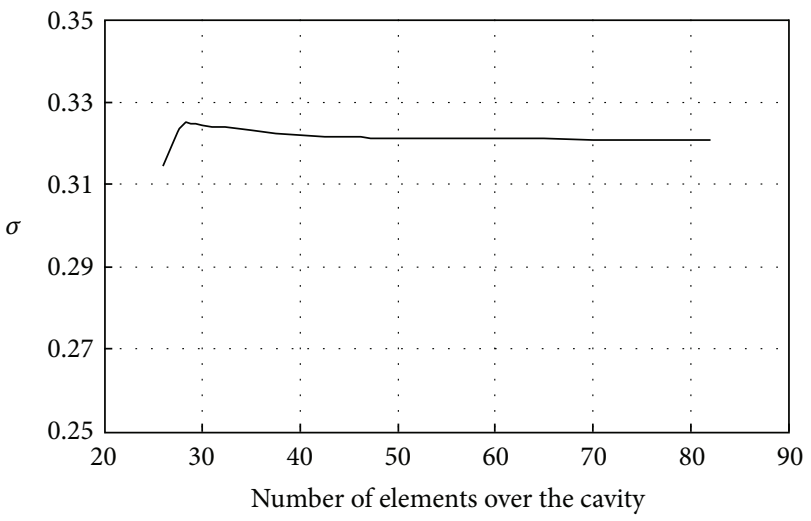

(b)

FIGURE 5: Convergence of the cavitation number with increasing the number of elements over (a) the cavitator (disc) and (b) the cavity.

is capable of making predictions for a wide range of cavity lengths.

Figure 7 displays supercavity length as a function of cavitation number $(\sigma)$ and Figure 8 displays the maximum thickness of cavity as a function of cavitation number. These figures indicate that the variation of cavity length and the maximum thickness of cavity are inversely related to the cavitation number. As shown in these two figures, the present BEM results agree with the analytical relation [2] and experimental data [2]. Remarkably, BEM may easily be implemented by a common PC for the analysis of flows with very small cavitation numbers (i.e., very large cavity lengths). However, Navier-Stokes based methods take much more time and extremely greater computational costs for a similar analysis. Further, while the analytical approach does not exhibit good results for large cavity lengths, the present BEM effectively predicts the cavitation number for these lengths.

8.2. Partial Cavitation. In Figure 9, the 3D view of partial cavitation as modeled by BEM on the blunt, hemispherical, and conical-head cylinders has been shown for $\sigma=0.3$. It is noticeable that, for a fixed cavitation number, the maximum cavity length occurs on the blunt-head cylinder and the minimum on the hemispherical-head cylinder. This matches expectations since when detachment point of the cavity has a fracture with a steeper slope on the body surface (such as a blunt-head cylinder), overshoot of flow will be greater 

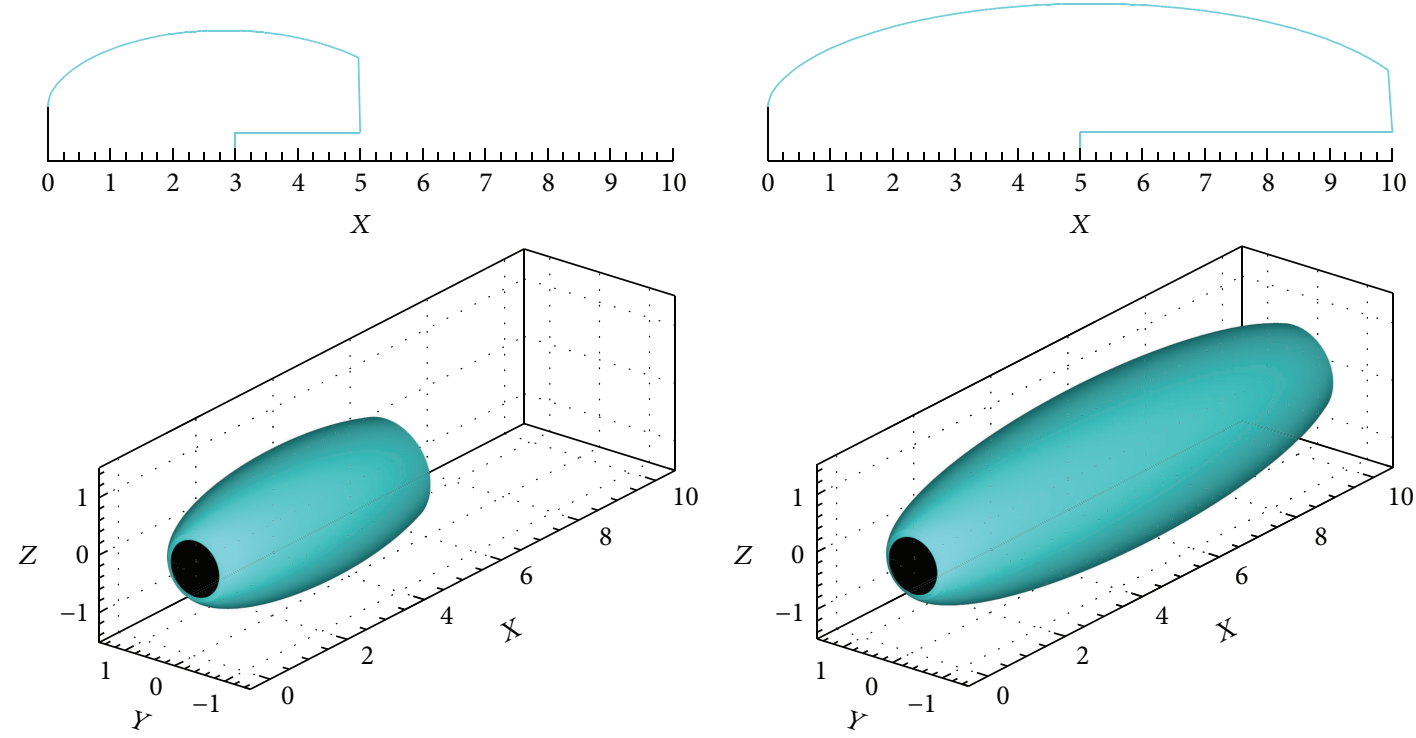

(a)

(b)

FIGURE 6: Converged cavity shapes over disc as predicted by BEM for (a) $l_{\text {cavity }} / D_{\text {disc }}=5, l_{\text {jet }} / l_{\text {cavity }}=0.5$, and $\sigma=0.272$ and (b) $l_{\text {cavity }} / D_{\text {disc }}=10$, $l_{\text {jet }} / l_{\text {cavity }}=0.5$, and $\sigma=0.149$.

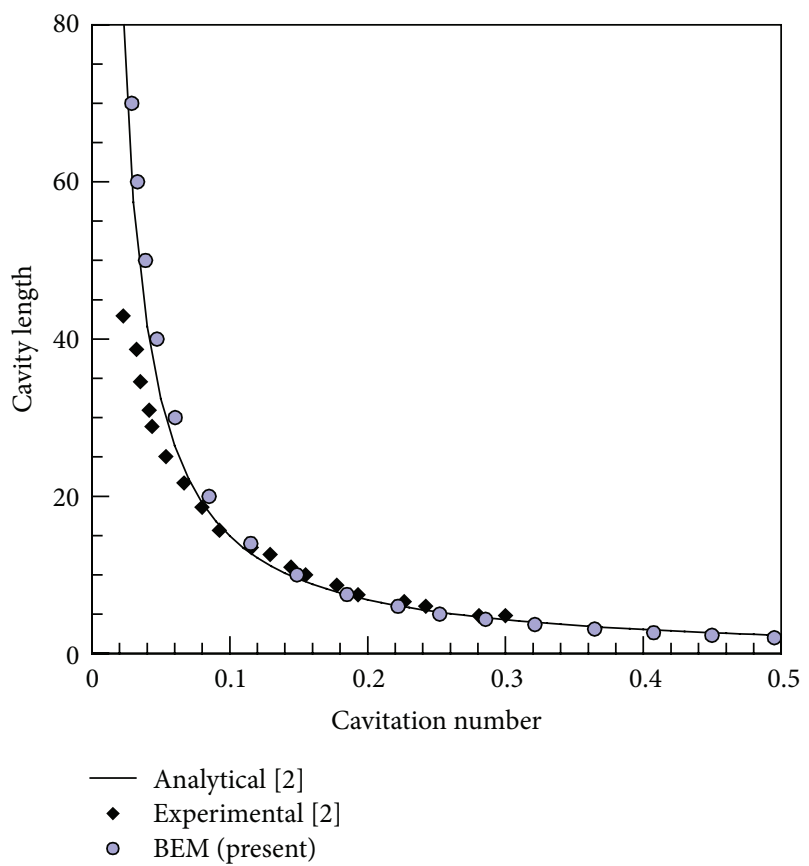

FIGURE 7: Prediction of cavity length $\left(l_{\text {cavity }} / D_{\text {disc }}\right)$ as a function of cavitation number $(\sigma)$ made by BEM in comparison with analytical relation and experimental data [2], for a disk cavitator.

and consequently cavity length will be larger. Figure 10 shows the distribution of pressure coefficient $\left(C_{P}\right)$ versus the length traveled on blunt, hemispherical, and conical-head cylinders for $\sigma=0.3$. The length is nondimensionalized by the diameter of the cavitator head. Evidently, BEM accurately

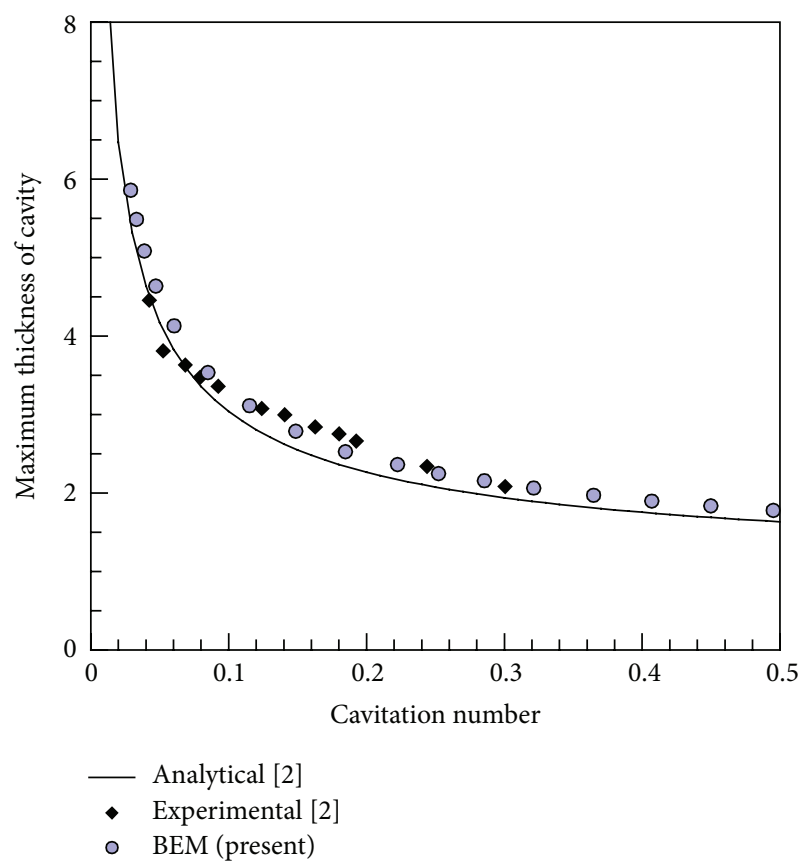

FIgURE 8: Prediction of the maximum thickness of cavity $\left(D_{\text {max,cavity }} / D_{\text {disc }}\right)$ as a function of cavitation number $(\sigma)$ made by BEM in comparison with analytical relation and experimental data [2], for a disk cavitator.

predicts the experimentally measured cavity length [26]. However, at the end of the cavity region where the cavity is closed on the cylinder body, the $C_{P}$ variation, as predicted by BEM with simple closure model, exhibits an unusual overshoot. This is due to the stagnation point that appears 


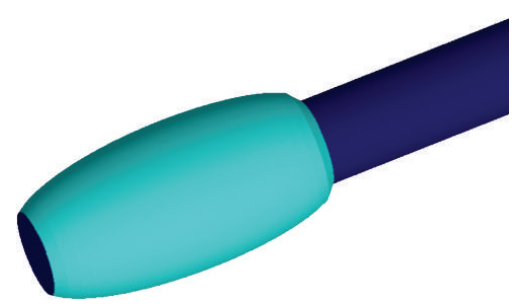

(a)

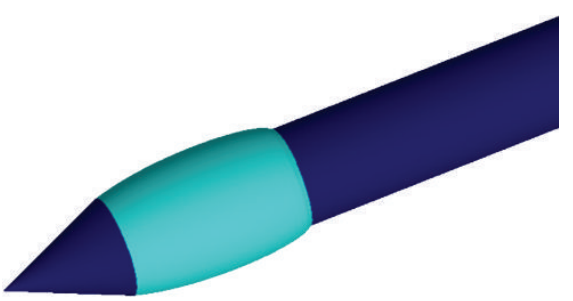

(b)

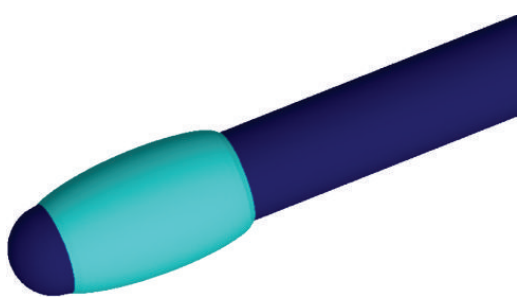

(c)

FiguRE 9: 3D view of partial cavitation as modeled by BEM on the (a) blunt, (b) conical, and (c) hemispherical cylinders for $\sigma=0.3\left(l_{\text {body }} / D_{c}=\right.$ 10).

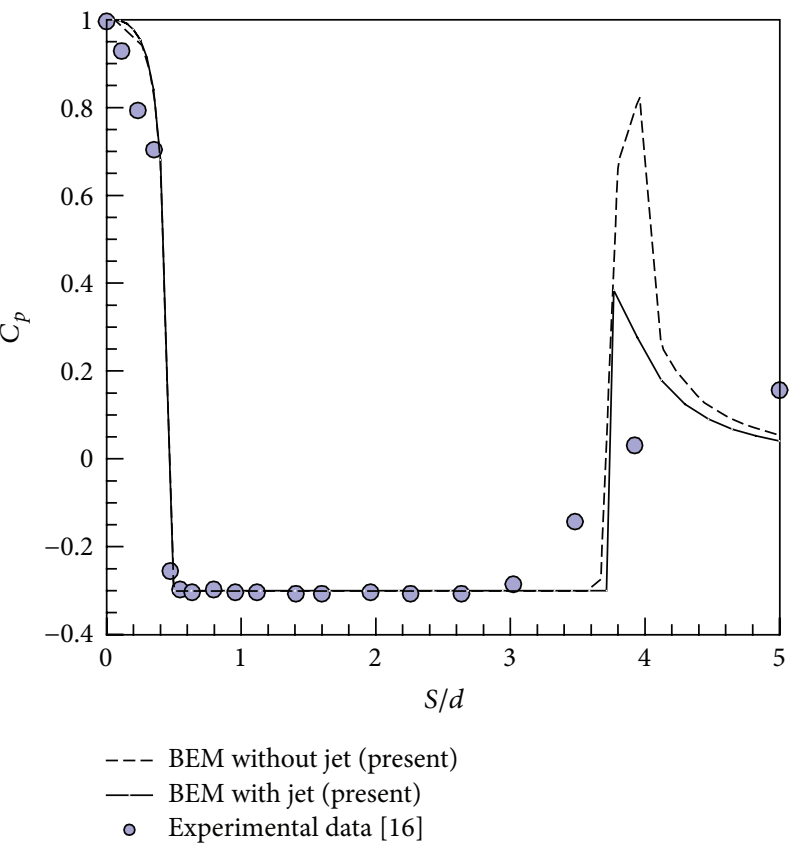

(a)

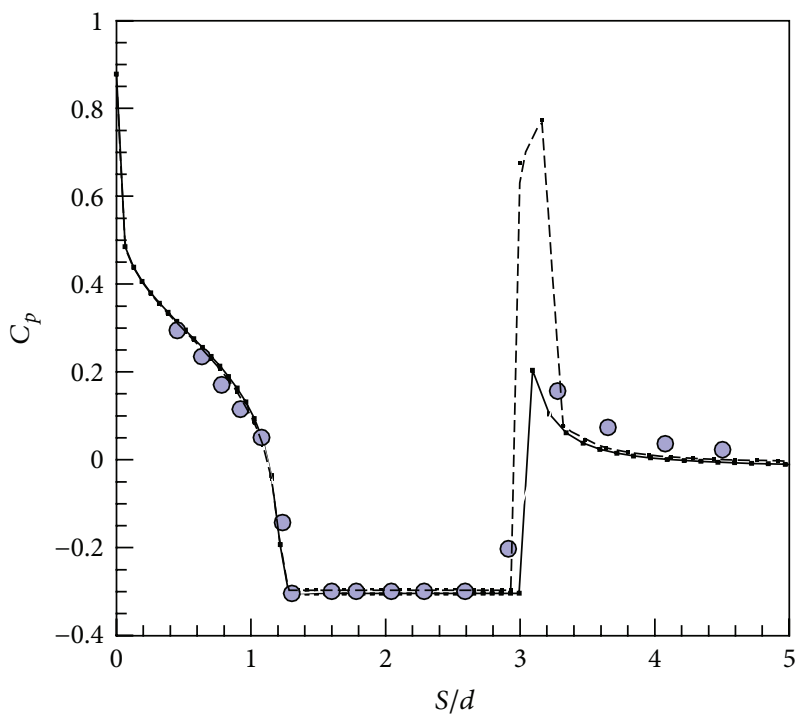

-- - BEM without jet (present)

- BEM with jet (present)

- Experimental data [26]

(b)

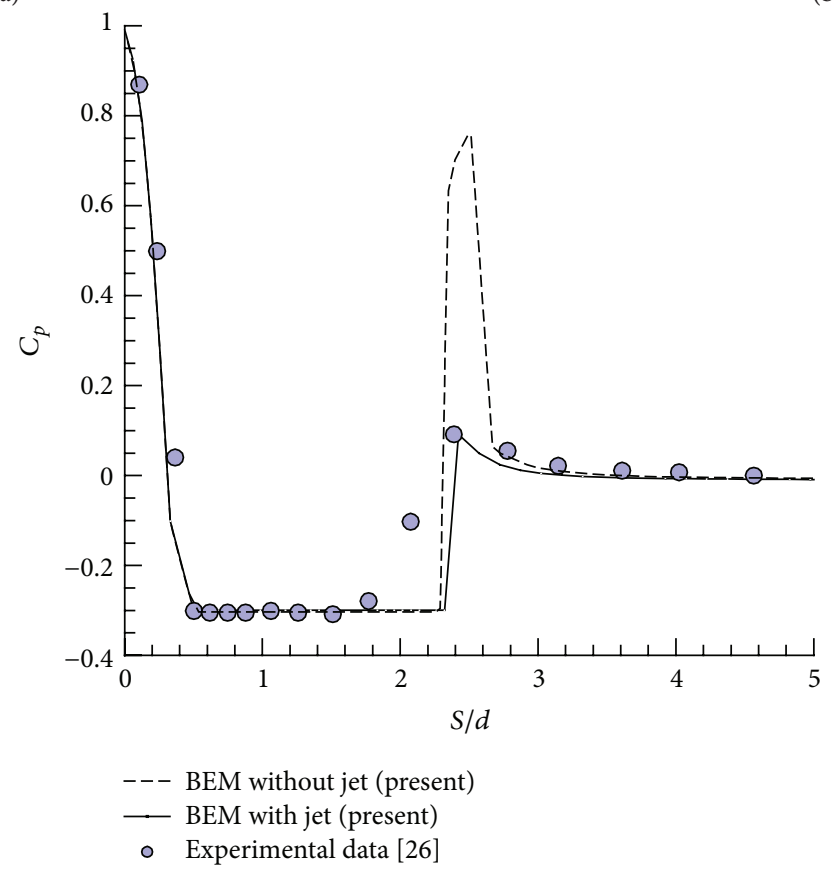

(c)

FIGURE 10: Distribution of pressure coefficient $\left(C_{P}\right)$ versus the length traveled on cylinders with blunt, hemispherical, and conical-head cylinders in comparison to experimental data [26], for $\sigma=0.3$. 


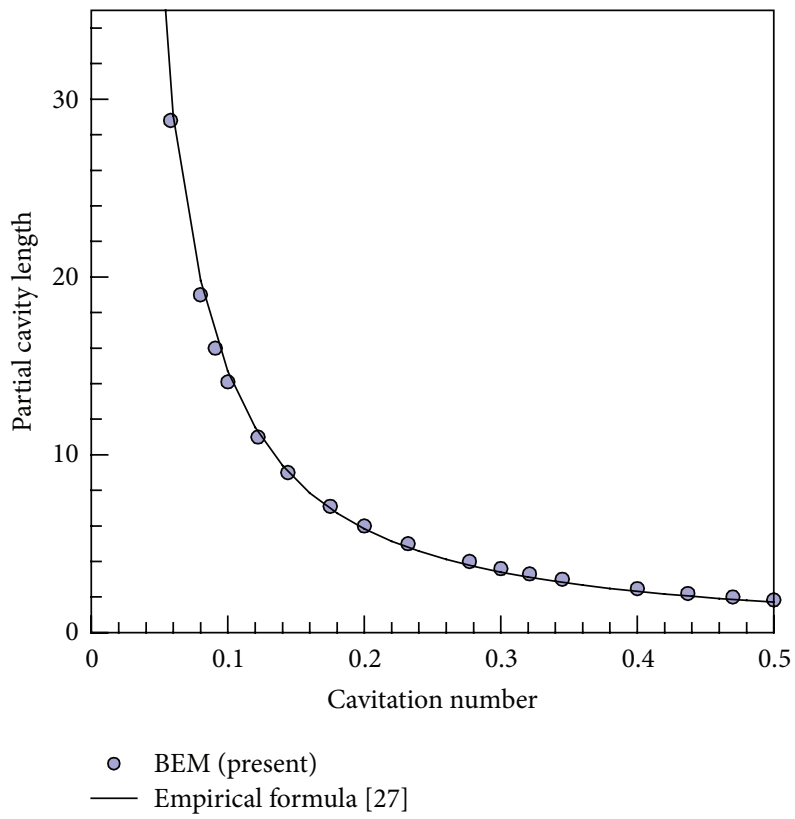

FIGURE 11: Prediction of partial cavity length $\left(l_{\text {cavity }} / D_{\text {head }}\right)$ as a function of cavitation number $(\sigma)$ made by BEM (using simple and reentrant jet model) in comparison to empirical formula [27], for a blunt-head cylinder $\left(l_{\text {body }} / D_{\text {head }}=40\right)$.

in the process of implementing the simple closure model. This defect, however, is attenuated in the reentrant jet model. BEM appropriately predicts the measurements for the rest of the cylinder. Figure 11 displays partial cavity length as a function of cavitation number $(\sigma)$. This figure indicates that the variation of partial cavity length is inversely related to the cavitation number. The present BEM results agree with the analytical relation [27]. In Figure 12, the effect of the chosen reentrant jet lengths on the solution results is shown. As shown, the cavitation number is essentially independent of the jet length in the range of $0<l_{\text {jet }} / l_{\text {cavity }}<75 \%$.

8.3. Quasi-3D Analysis. Figures 13 and 14 serve to compare the results of quasi-3D BEM with those of axisymmetric and $3 \mathrm{D}$ simulations based on a multiphase mixture method (using commercial CFD code: Fluent 6.3.26) for $\sigma=0.2$, 0.3 , respectively. The shape in question is a cylinder with an elliptical head and the aspect ratio of $R_{\max } / R_{\min }=1.2$. The smaller diameter of the elliptical head is located along the $y$-axis and the larger diameter along the $x$-axis. In addition, the cylinder is set to be 20 units long (nondimensionalized by the smaller diameter of ellipsis). It is noticeable that the quasi-3D BEM results are good approximations to the results from the 3D simulations of the mixture method. The cavity length in quasi-3D BEM is slightly longer than the cavity length of 3D mixture method. This difference is owing to the assumption that, in BEM, flow is potential and has a fixed velocity on the cavity. Note that, for smaller $\sigma$ s, these two assumptions match the real data more closely. Consequently, the results from the two methods are closer to each other in $\sigma=0.2$. In the worst-case scenario, the quasi-3D BEM prediction for the cavity length exceeds the prediction of

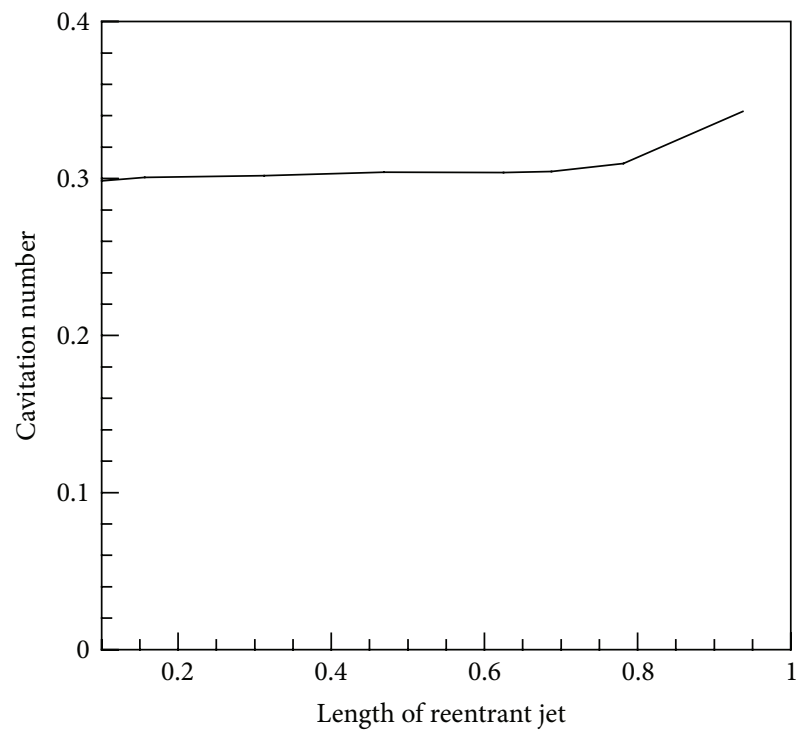

FIGURE 12: Effect of the chosen reentrant jet lengths on the solution results.

the $3 \mathrm{D}$ mixture method no more than $15 \%$. Figure 15 is a demonstration of the 3D view of partial cavitating flow as modeled by the above methods, for $\sigma=0.3$. A comparison of the $3 \mathrm{D}$ views that resulted from the two methods corroborates the capability of BEM for making quasi-3D predictions of cavitating flows. Figure 16 shows the variation of maximum length of partial cavity (nondimensionalized by the smaller diameter of elliptical head) versus cavitation number for various aspect ratios. The figure shows that, at 

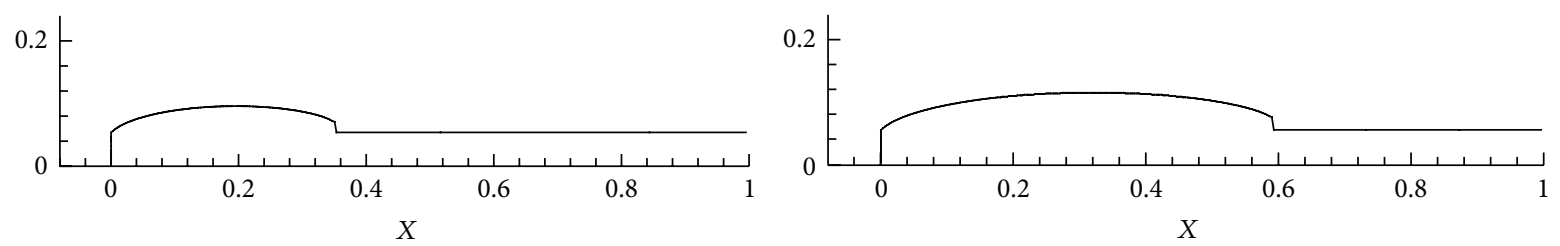

(a)
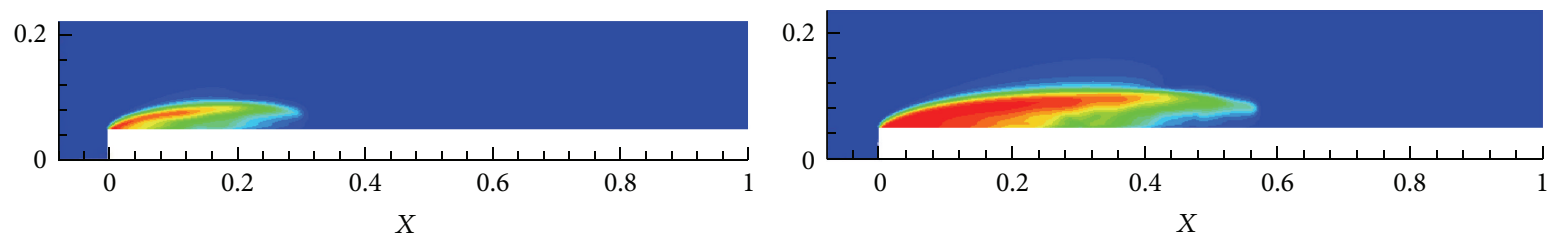

(b)
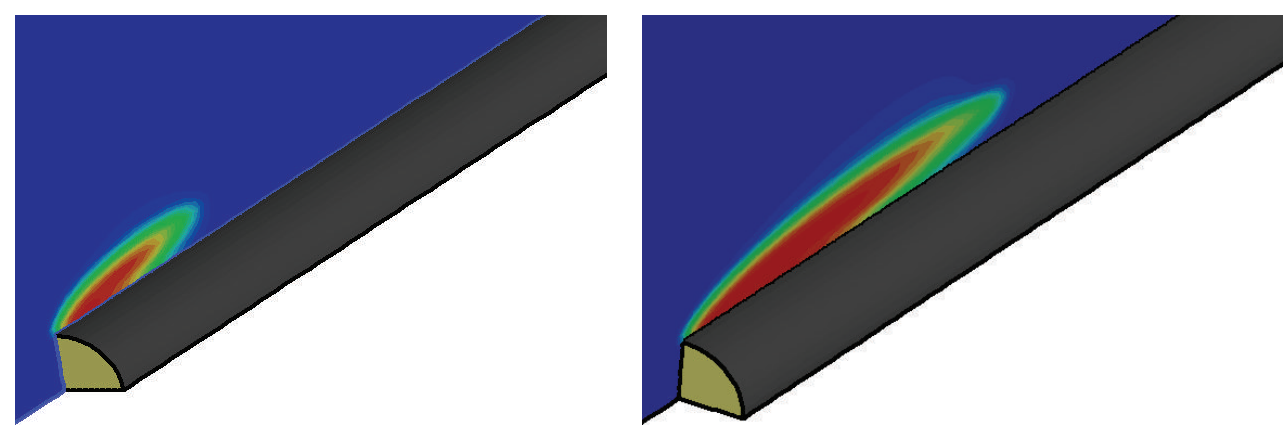

(c)

FIGURE 13: View of partial cavity over a blunt cylinder for the smaller diameter of the elliptical cross section $\left(R_{\max }=0.5\right.$ along the $y$-axis $)$ modeled by (a) quasi-3D BEM, (b) axisymmetric Navier-Stokes, and (c) 3D Navier-Stokes for left hand $\sigma=0.3$ and right hand $\sigma=0.2$.

TABLE 1: A comparison of meshing and convergency between BEM and Navier-Stokes method.

\begin{tabular}{lccc}
\hline Model & Dimension & Number of elements (meshes) & Time of convergency (minute) \\
\hline \multirow{2}{*}{ BEM } & Axisymmetric & $\sim 200$ & $\sim 3$ \\
& Quasi-3D & $\sim 2400$ & $\sim 25$ \\
\hline \multirow{2}{*}{ Navier-Stokes } & Axisymmetric & $\sim 90000$ & $\sim 400$ \\
& 3D & $\sim 600000$ & $\sim 2100$ \\
\hline
\end{tabular}

greater aspect ratios, the maximum length of partial cavity is larger.

In addition, to illustrate the ability of the quasi-3D BEM algorithm, the simulation of cavitating flow around elliptical cross section bodies with hemispherical, conical, and semiconical cavitator has been performed and shown in Figure 17, for $\sigma=0.2$ and $R_{\max } / R_{\min }=1.2$. The angle of conical and semiconical cavitator in smaller diameter of elliptical cross section is 22.5 degrees. The figure shows that, for a fixed cavitation number, the maximum cavity length occurs on the conical-head cylinder $\left(L_{\max , \text { cavity }} / D_{\min , c}=\right.$ 3.04 ) and the minimum on the semi-conical-head cylinder $\left(L_{\text {max }, \text { cavity }} / D_{\min , c}=2.37\right)$.

8.4. A Comparison of Meshing and Convergency. In Table 1, the two methods of BEM and Navier-Stokes (multiphase mixture method) are compared in terms of the number of elements (meshes) and convergency. Obviously, the two methods were implemented using the same PC. As shown, the elements in axisymmetric BEM are 450 times fewer than the meshes in the axisymmetric Navier-Stokes method and at least 250 times fewer than the meshes in the 3D Navier-Stokes method. The remarkable difference between BEM and Navier-Stokes method in terms of the number of elements (meshes) leads to such a considerable difference in convergency that BEM converges about 100 times faster than the Navier-Stokes method.

\section{Conclusions}

A BEM analysis of super- and partial cavitating flows around axisymmetric bodies is performed and two models of the 

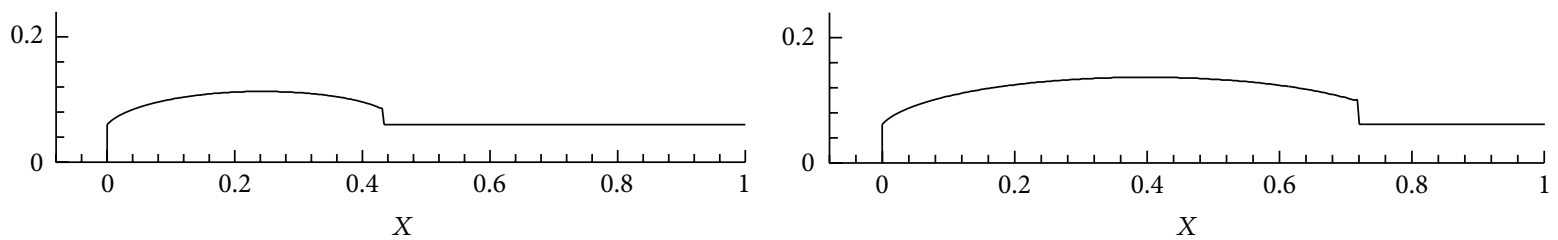

(a)
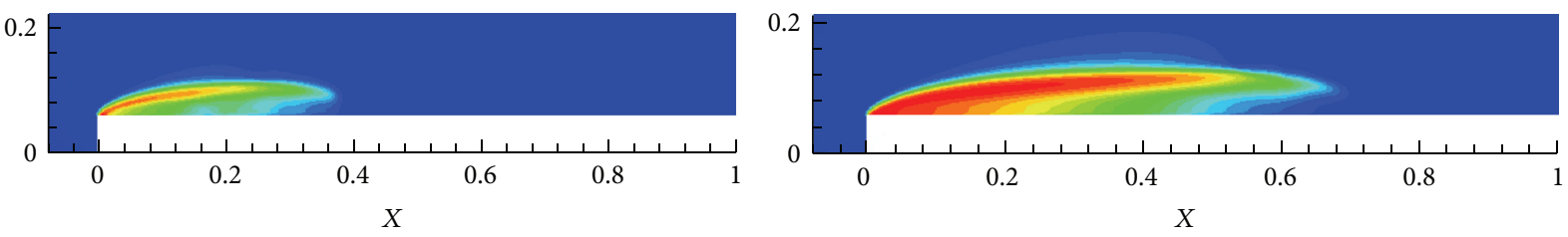

(b)
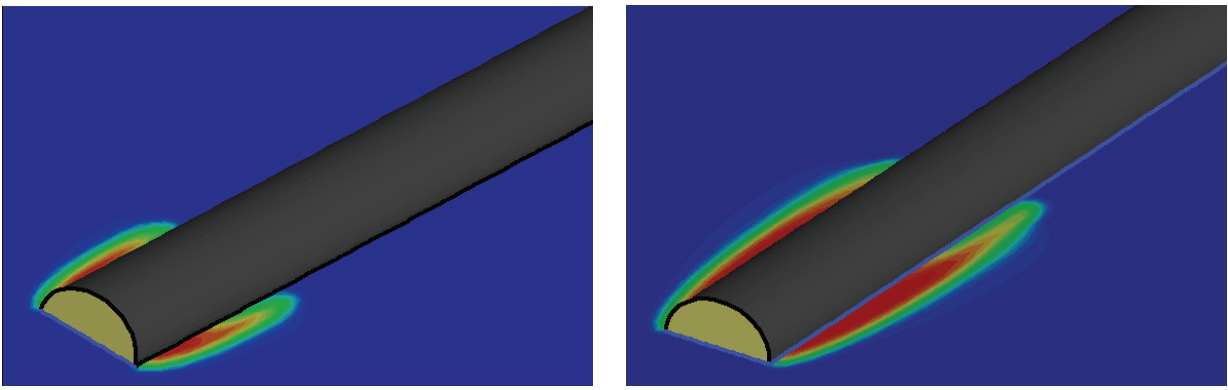

(c)

FIGURE 14: View of partial cavity over a blunt-head cylinder for the larger diameter of the elliptical cross section $\left(R_{\max }=0.6\right.$ along the $x$-axis $)$ modeled by (a) quasi-3D BEM, (b) axisymmetric Navier-Stokes, and (c) 3D Navier-Stokes for left hand $\sigma=0.3$ and right hand $\sigma=0.2$.

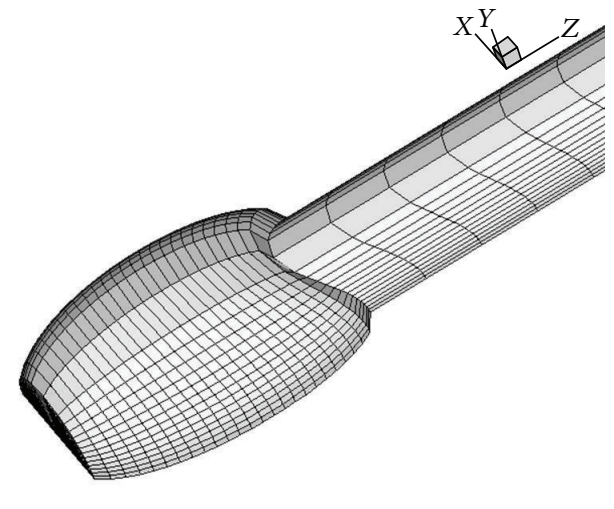

(a)

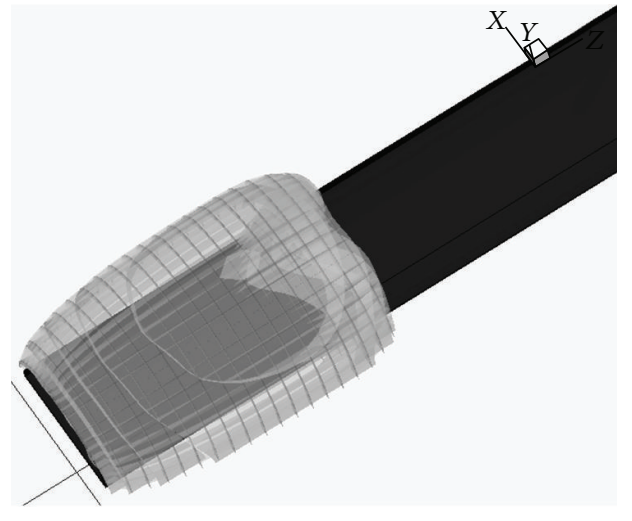

(b)

FIGURE 15: 3D view of partial cavitation on the blunt cylinder for $\sigma=0.3\left(l_{\text {body }} / D_{\min , c}=10\right)$, modeled by (a) quasi-3D BEM and (b) 3D Navier-Stokes for $\sigma=0.3$.

cavity closure (simple and reentrant jet models) are compared. Results show that the reentrant jet model is more adapted with experimental data at the closure zone of the cavity in pressure coefficient distribution results. Also, a simple algorithm is introduced to model quasi-3D cavitating flows (cylinders with elliptical head) using this axisymmetric code. The results show that using this algorithm is interesting because of its simplification, lower computational cost, and good agreement with numerical CFD results. The present analysis yields the relatively accurate results at minimal computational and time cost, whereas the other numerical methods involve greater complication and higher cost. 


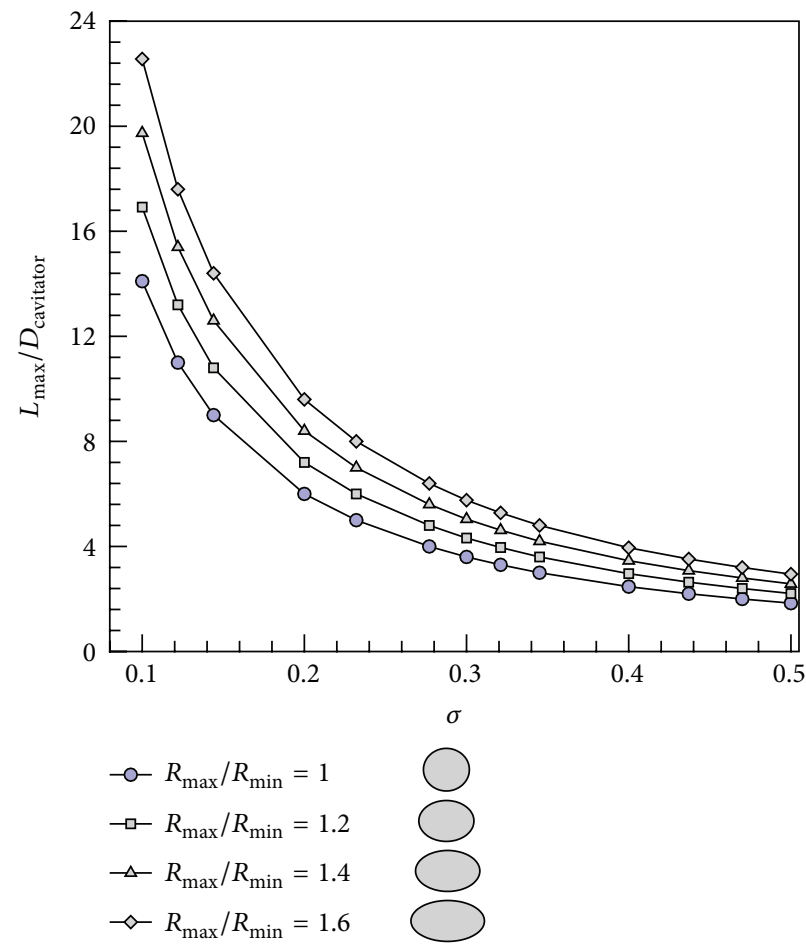

FIGURE 16: Variation of maximum length of the cavity over elliptical-head cylinder for various aspect ratios versus cavitation number modeled by quasi-3D BEM.

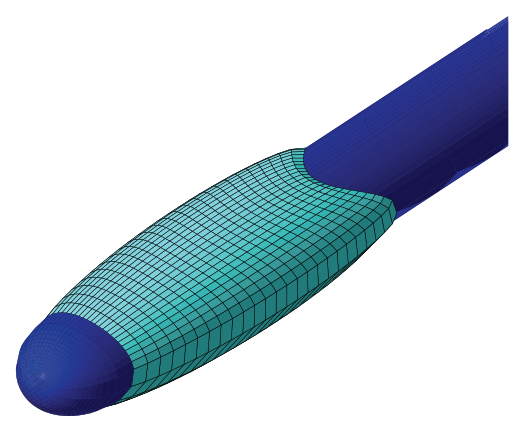

(a) Hemispherical head

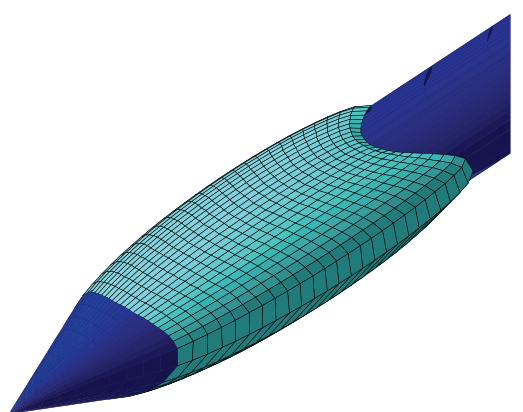

(b) Conical

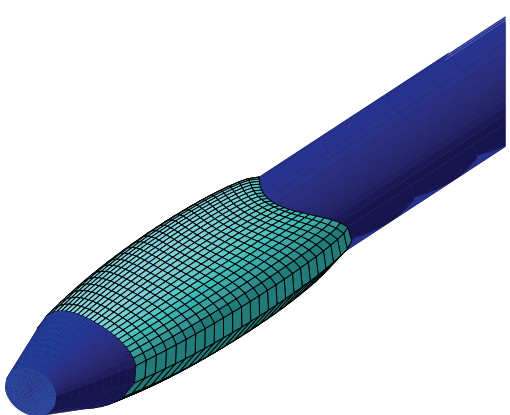

(c) Semiconical

FiguRE 17: 3D view of partial cavitation on the various cavitator with elliptical cross section for $\sigma=0.2$ and $l_{\text {body }} / D_{\min , c}=20$ and $R_{\max } / R_{\min }=$ 1.2 , modeled by quasi-3D BEM algorithm.

\section{Competing Interests}

The authors declare that there are no competing interests regarding the publication of this paper.

\section{References}

[1] E. A. Euteneuer, Further studies into the dynamics of a supercavitating torpedo [Ph.D. thesis], Department of Aerospace Engineering and Mechanics, University of Minnesota, Minneapolis, Minn, USA, 2003.

[2] J. P. Franc and J. M. Michel, Fundamental of Cavitation, Kluwer Academic, Amsterdam, Netherlands, 2004.
[3] K. R. Labertaux and S. L. Ceccio, "Partial cavity flows (part 1), cavities forming on models without span wise variation," Journal of Fluid Mechanics, vol. 431, pp. 1-41, 2001.

[4] S. A. Kinnas and N. E. Fine, "A numerical nonlinear analysis of the flow around two- and three-dimensional partially cavitating hydrofoils," Journal of Fluid Mechanics, vol. 254, pp. 151-181, 1993.

[5] A. G. Efros, "Hydrodynamic theory of two-dimensional flow with cavitation," Doklady Akademii Nauk SSSR, vol. 51, pp. 267270, 1946.

[6] D. Gilbarg and J. Serrin, "Free boundaries and jets in the theory of cavitation," Journal of Mathematics and Physics, vol. 29, no. 1-4, pp. 1-12, 1950. 
[7] M. P. Tulin, "Steady two-dimensional cavity flows about slender bodies," Tech. Rep. DTMB 834, Navy Department, Washington, DC, USA, 1953.

[8] M. P. Tulin, "Supercavitating flows—small perturbation theory," Journal of Ship Research, vol. 7, no. 3, pp. 16-37, 1963.

[9] J. A. Guerst, Linearized theory of two-dimensional cavity flows [Ph.D. thesis], Delft University of Technology, Delft, Netherlands, 1961.

[10] J. L. Hess and A. M. O. Smith, "Calculation of potential flow about arbitrary three-dimensional bodies," Progress in Aeronautical Science, vol. 8, pp. 1-138, 1966.

[11] J. S. Uhlman, "The surface singularity method applied to partially cavitating hydrofoils," Journal of Ship Research, vol. 31, no. 2, pp. 107-124, 1987.

[12] J. S. Uhlman, "The surface singularity or boundary integral method applied to supercavitating hydrofoils," Journal of Ship Research, vol. 33, no. 1, pp. 16-20, 1989.

[13] S. A. Kinnas and N. E. Fine, "Non-linear analysis of the flow around partially or super-cavitating hydrofoils by a potential based panel method," in Boundary Integral Methods: Theory and Applications Proceedings of the IABEM Symposium Rome, Italy, October 15-19, 1990, pp. 289-300, Springer, Berlin, Germany, 1991.

[14] G. Birkhoff and E. H. Zarantonello, Jets, Wakes and Cavities, Academic Press, New York, NY, USA, 1957.

[15] D. Gilbarg, "Jets and cavities," in Handbuch der Physik, vol. 9, Springer, Berlin, Germany, 1960.

[16] C. Pellone and A. Rowe, "Supercavitating hydrofoils in nonlinear theory," in Proceedings of the 3rd International Conference on Numerical Ship Hydrodynamics, Paris, France, 1981.

[17] N. E. Fine and S. A. Kinnas, "A boundary element method for the analysis of the flow around 3-D cavitating hydrofoils," Journal of Ship Research, vol. 37, no. 3, pp. 213-224, 1993.

[18] V. B. Vaz, Modelling of sheet cavitation on hydrofoils and marine propellers using boundary element methods [Ph.D. thesis], Universidade T'ecnica de Lisboa, Lisbon, Portugal, 2005.

[19] J. S. Uhlman, "A note on the development of a nonlinear axisymmetric reentrant jet cavitation model," Journal of Ship Research, vol. 50, no. 3, pp. 259-267, 2006.

[20] N. M. Nouri, M. Moghimi, and S. M. H. Mirsaeedi, "Numerical simulation of unsteady cavitating flow over a disc," Proceedings of the Institution of Mechanical Engineers, Part C: Journal of Mechanical Engineering Science, vol. 224, no. 6, pp. 1245-1253, 2010.

[21] I. Rashidi, M. Passandideh-Fard, and M. Pasandideh-Fard, "The optimum design of a cavitator for high-speed axisymmetric bodies in partially cavitating flows," Journal of Fluids Engineering, Transactions of the ASME, vol. 135, no. 1, Article ID 011301, 2013.

[22] M. Callenaere, J.-P. Franc, J.-M. Michel, and M. Riondet, "The cavitation instability induced by the development of a re-entrant jet," Journal of Fluid Mechanics, vol. 444, pp. 223-256, 2001.

[23] S. Washio, S. Takashashi, and S. Yoshimori, "Study on cavitation starting at the point of separation on a smooth wall in hydraulic oil flow," Proceedings of the Institution of Mechanical Engineers, Part C: Journal of Mechanical Engineering Science, vol. 217, no. 6, pp. 619-630, 2003.

[24] H.-F. Peng, M. Cui, and X.-W. Gao, "A boundary element method without internal cells for solving viscous flow problems," Engineering Analysis with Boundary Elements, vol. 37, no. 2, pp. 293-300, 2013.
[25] M. Manninen, V. Taivassalo, and S. Kallio, On the Mixture Model for Multiphase Flow, Technical Research Centre of Finland, VTT Publications 288. 67, 1996.

[26] H. Rouse and J. S. Mc Nown, "Cavitation and pressure distribution," in Head Forms at Zero angle of Yaw, vol. 32 of Studies in Engineering, State University of Iowa, 1948.

[27] M. L. Billet and D. S. Weir, "The effect of gas diffusion on the flow coefficient for a ventilated cavity," Journal of Fluids Engineering, vol. 97, no. 4, pp. 501-505, 1975. 


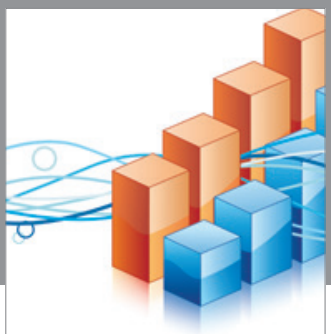

Advances in

Operations Research

vatem alat4

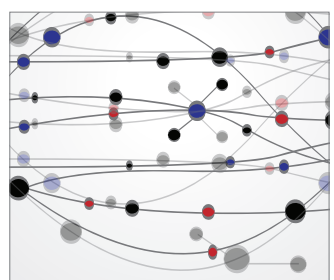

\section{The Scientific} World Journal
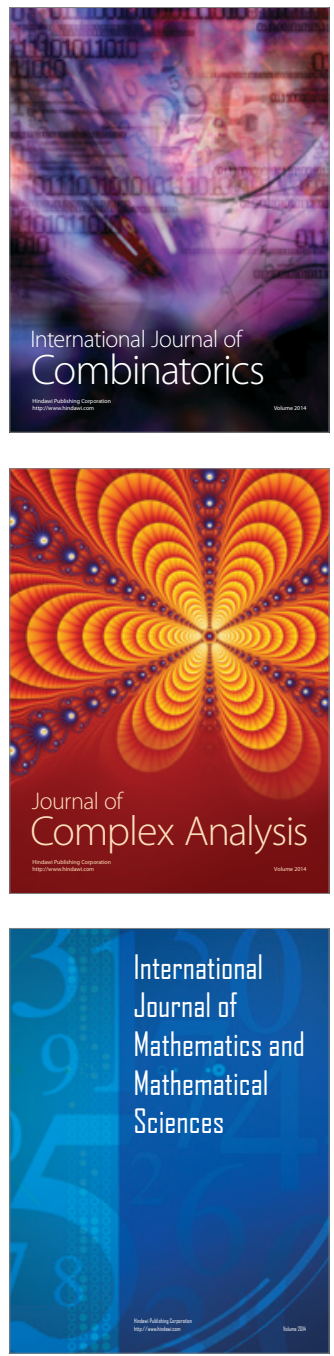
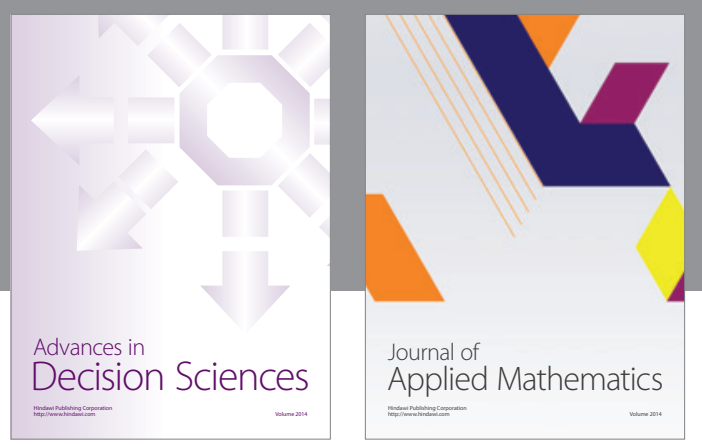

Algebra

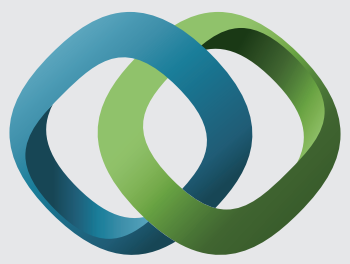

\section{Hindawi}

Submit your manuscripts at

http://www.hindawi.com
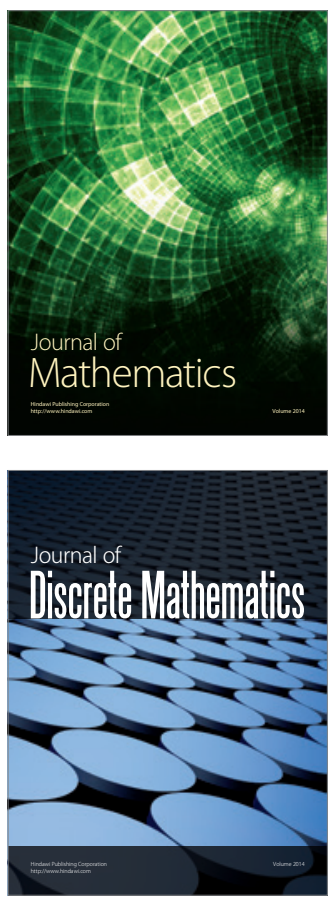

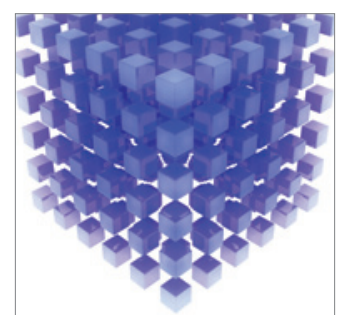

Mathematical Problems in Engineering
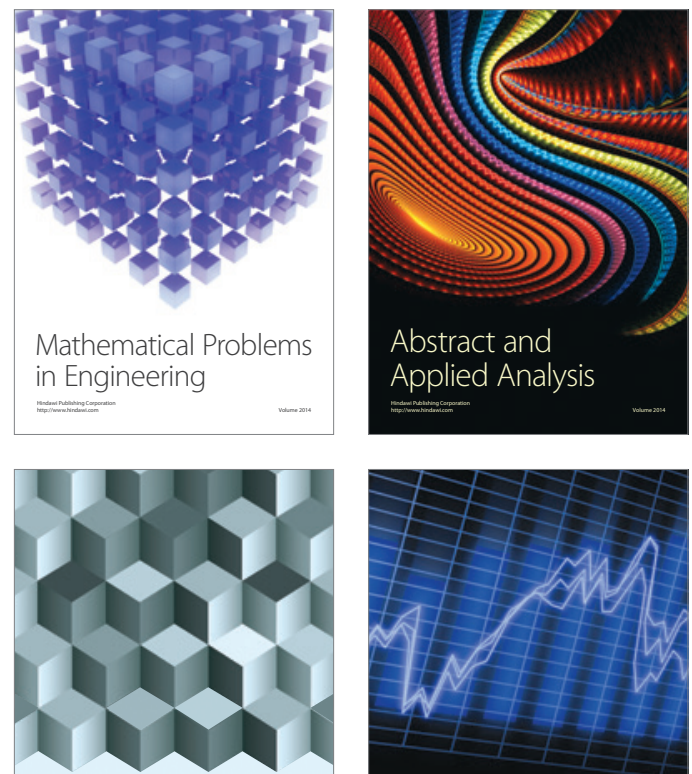

Journal of

Function Spaces

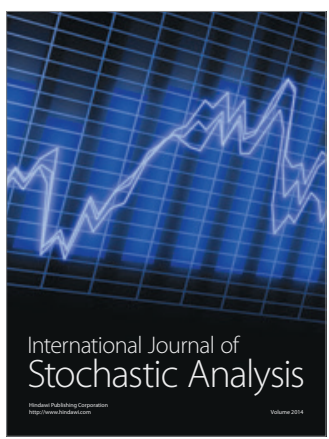

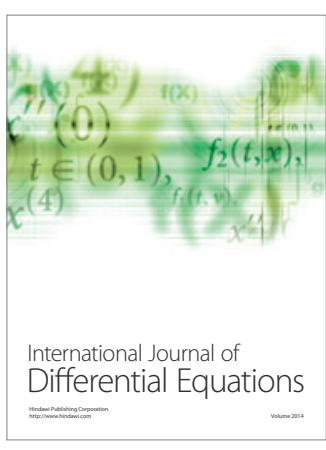
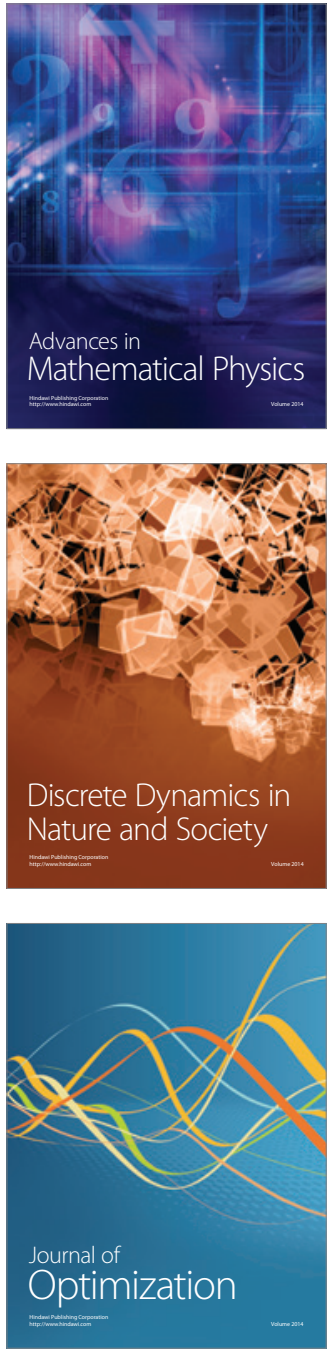